\title{
Connected functional working spaces: a framework for the teaching and learning of functions at upper secondary level
}

\author{
Tran Kiem Minh, College of Education, Hue University, Vietnam \\ Jean-Baptiste Lagrange, LDAR University Paris -Diderot and University of Reims, France
}

\begin{abstract}
This paper aims at contributing to remedy the narrow treatment of functions at upper secondary level. Assuming that students make sense of functions by working on functional situations in distinctive settings, we propose to consider functional working spaces inspired by geometrical working spaces. We analyse a classroom situation based on a geometric optimization problem pointing out that no working space has been prepared by the teacher for students' tasks outside algebra. We specify a dynamic geometry space, a measure space and an algebra space, with artefacts in each space and means for connecting these provided by Casyopée. The question at stake is then the functionality of this framework for implementing and analyzing classroom situations and for analyzing students' and teachers' evolution concerning functions, in terms of geneses relative to each space.
\end{abstract}

Keywords Functions, Working spaces, Casyopée, Genesis, Connected functional working spaces.

The final publication is available at Springer via http://dx.doi.org//10.1007/s11858-016-0774-z

\section{Introduction}

This paper is about the teaching of functions at upper secondary level. We are working in separate countries, but in the same research group. This "Casyopée" group brings together teachers and researchers. Its main concern is that, although both at an epistemological and at a cognitive level, functions make sense because of their occurrence in many dissimilar settings, teaching actually favors "algebraic" representations, that is to say formulas and the associated graphs and tables, and often senseless manipulation, providing students poor understanding and abilities. Our group tries to implement classroom situations, tools and resources and a theoretical basis to remediate this narrow treatment of functions by upper secondary teaching.

The motivation of this paper is that, among the theoretical frameworks that our group considered before, no one took into consideration the fact that, to be fully considered by students and teachers, each setting where functions can make sense has to be thought of as a particular space allowing specific scientific work and specific conceptualisations. We are influenced by Kuzniak (2013), who presents geometrical working spaces as a way to avoid misunderstandings in geometrical education, for instance with regard to how students should reason, developing their spatial intuition and ability with instruments, or rejecting these in favor of formal deduction. Like geometrical working spaces, considering functional working spaces should allow teachers and students to work on functions in various spaces, including spaces where functions are experienced without algebraic formalization, avoiding the predominance of a working space restricted to algebraic representations and manipulations. As with geometrical working spaces, working in a specific functional space should allow working on functions with specific instruments and under control of specific rules. The hypothesis in this paper is that adequate teaching situations should organize students' work in various non-algebraic and algebraic working spaces, and allow connections between these. 
We first develop our analysis of current curricula, taking the example of our countries. Then, drawing from the work of other researchers, we analyse how current strategies orient students' activity to a restricted working space. This helps to specify three working spaces relevant for a class of problems, consistent with our hypothesis. We show by another example how the design of a classroom situation can take advantage of considering these spaces. This design involves a resource built by the Casyopée group, the Casyopée software, and we show how this environment provides specific artefacts for each space, and helps make connections between spaces.

The question then is how students and teachers come to understand the affordances provided by each of these spaces respectively for learning and for teaching, that is to say, in Kuzniak's (2013) words, how they build suitable personal functional working spaces, a process that we name genesis ${ }^{1}$. Our hypothesis is that, because this understanding comes along with the conceptualization of functions, a genesis necessarily occurs in the long term. We carried out studies about students and teachers over two years, concerning functions and Casyopée, reported respectively by Minh (2012a) and Lagrange (2011). In this paper, we take advantage of both these studies and of the identification of functional working spaces, in order to understand how personal functional spaces are built by students and teachers.

\section{Functions, curricula and classroom implementation}

The functional perspective on the teaching of algebra is seen by curricula makers as an effective approach to consolidate post middle school students' algebraic knowledge and to prepare them for calculus. The use of technology, especially graphical and dynamic geometry packages, is encouraged in an exploratory approach to functions. Kieran (2007) depicts these programs of study as oriented towards solving realistic problems and towards multirepresentational activities, allowing for an algebraic content that is less manipulation oriented and thus shifting away from traditional skills of algebra.

Lagrange and Psycharis (2014), analyzing students' activities on functions from different perspectives, identified three important challenges for students: to consider functional dependencies, that is to say something depending on another, as particular covariation, to understand the idea of independent variable, and to make sense of functional symbolism. The first two challenges point towards activities where a functional dependency is studied in various representations or models. The third one implies linking dependency with various algebraic forms: notation $f(\ldots)$, domain of existence, role of independent variable and of parameters in the formula...

Considering the curricula in our countries as examples representative of dissimilar cultures, we illustrate how precisely functions appear in programs of studies. In Vietnam, Nguyen (in Halbert et al., 2013) stresses that $60 \%$ of the program deals with functions and that it is organized around this domain. The ideas of functional dependency and of variations of functions ${ }^{2}$, beyond mere correspondence, are introduced early (respectively $7^{\text {th }}$ and $9^{\text {th }}$ grade). After $10^{\text {th }}$ grade, the curriculum is more oriented towards classical study of functions. The main

${ }^{1} \mathrm{We}$ are aware that 'genesis' is used for denoting, in MWS, processes connecting the epistemological and the cognitive planes in a space. However, the way we use this word is consistent with the theoretical construct of 'instrumental genesis' (Lagrange, 1999), a basis of the work we carried out for more than 15 years. Confronting these two conceptions is a promising perspective.

${ }^{2}$ Studying variations of a continuous function is for instance proving that it is decreasing on some interval and increasing on another adjacent interval, and thus that it has a minimum. See examples in the teaching situations above. 
types of tasks proposed by textbooks are also classical. Only one type of tasks deals also with another domain: optimizing a geometrical quantity. Nguyen Thi Nga (2011) gives complementary insight, stressing how this program demands that teachers carry out classroom mathematically meaningful interdisciplinary activities. However she points out that actual tasks proposed to students, even when they involve extra-mathematical situations, are rather applications of already taught mathematics.

In France, before $10^{\text {th }}$ grade, functions are included in a "field" dealing with data management in which proportionality and statistics have an important place. At this stage, functions are not algebraic. Algebra deals with equations and inequations. At $10^{\text {th }}$ grade there is a strong emphasis on the functional perspective mentioned above: half of the program is made of a "field" of functions, the other half being left to geometry and probabilities. The program insists on problem solving involving situations in various domains, mathematical and extra-mathematical. MEN/DGESCO-IGEN (2013) is an official document accompanying this program of study that stipulates competencies to be developed by students. Proficiencies like "researching", "representing" and "calculating" are well specified by relevant sub-competencies. In contrast, the proficiency "modelling" is poorly described as the ability "to translate real world situations into the mathematical language [sic]". Thus, as in Vietnam, tasks involving extra-mathematical situations give priority to mathematical symbolism. At $11^{\text {th }}$ and $12^{\text {th }}$ grade, the curriculum is classically oriented towards calculus ${ }^{3}$.

These two examples of curricula show that the emphasis on functions in some parts of programs of study does not go together with activities related to functional dependencies through which students could access the idea of function and associated symbolism, and meet the above mentioned challenges. Focusing on real world situations "translated into into the mathematical language," the curricula actually favor application of classical "algebraic" methods. Later, at $11^{\text {th }}$ and $12^{\text {th }}$ grades, these methods evolve towards classical calculus, again not involving deep understanding of functions. This is what we called above the narrow treatment of functions by upper secondary teaching.

Above, we mentioned one type of task in Vietnam associating function to another domain: optimizing a geometrical quantity. This type of task is also encouraged in France at $10^{\text {th }}$ grade. We draw an illustration of the narrow treatment of functions at the level of the classroom, from an article by Robert and Vandebrouk (2014) aiming at studying the influence of teachers' decisions regarding tasks and classroom implementation upon students' activity. The authors analyse classroom situations and we select one that implements a task belonging to the above mentioned type. They say (p. 268) that this situation is representative of the teacher's usual practice when dealing with functions and technology. From information provided by this article, figure 1 presents the geometrical figure at stake, the sub-tasks for students, and some complementary data with regard to the teacher's expectation. The figure made of the square DEFG and the triangle FBA is supposed to represent a shop-ensign, a weak response to the curriculum's demand of "real life situation".

Robert and Vandebrouck (2014) report on the work in the class, focusing on two pairs of students. The account gives evidence of a gap between what actually happened and the teacher's expectations. The first pair of students is weak. They spend 30mn on constructing the figure and achieve it only with the help of the teacher. They explore with difficulty, finding a minimum again thanks to the teacher, but cannot use the trace of a point. They do not understand what "calculating the sum of areas as a function of $x$ " means. During the last $10 \mathrm{mn}$, the teacher explains to the whole class how to "complete the square" and students copy what is written on the

\footnotetext{
${ }^{3}$ The current programs of study and accompanying documents in France can be found at http://eduscol.education.fr/
} 
blackboard. The other pair is high achieving. They understand correctly the tasks in dynamic geometry, using the trace to complete the exploration as indicated by the teacher. They make the link between the trace and numerical exploration, but they do not see the trace as a graph of a function. After that, they do not understand the purpose of an algebraic expression. Nevertheless, they obtain this expression, but they do not know how to use it. Like the other pair, they follow the teachers' explanation about "completing the square". These students had much less difficulty as compared to the other pair to perform sub-tasks, but for them also the sub-tasks are not connected because the idea of function that should make the connection is not present.

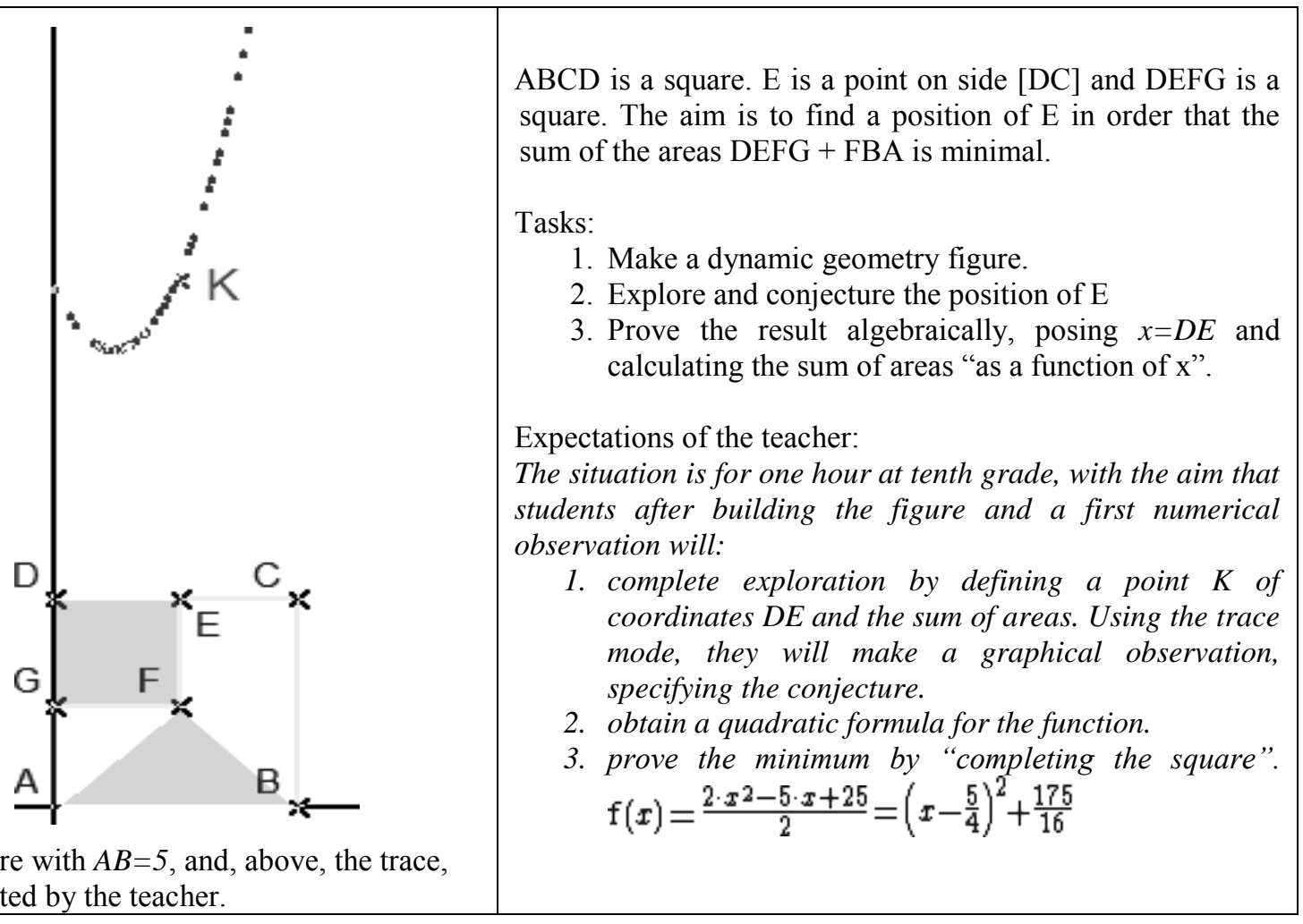

Fig. 1 The "shop-ensign" scenario

In their analysis, Robert \& Vandebrouck (2014) note that the decision of directing students to use " $x=D E$ " implies that students should concentrate on algebraic work, creating a gap with activity in dynamic geometry. Our analysis is complementary. First, in sub-task 1, we see what we call an underestimation by the teacher of instrumental needs of the situation in dynamic geometry. It means that students' activity expected by the teacher presupposes an understanding of complex features of dynamic geometry software, in connection with mathematical understanding. Here students have to be aware of a distinction between fixed point, free point and free point on an object, and understand what a dependent point is. This awareness is linked to the idea of function that students are supposed to approach through this task. Very often beginners in dynamic geometry confuse free point in the plane and free point on object and have no idea of dependent point ${ }^{4}$. It means here that

${ }^{4}$ We submitted a dynamic geometry figure with $\mathrm{A}$ a fixed point, $\mathrm{C}$ a free point, and $\mathrm{B}$ and $\mathrm{D}$ constructed in order that $\mathrm{ABCD}$ is a rectangle with sides parallel to the axes, to 34 students in a $10^{\text {th }}$ grade class. The students had basic knowledge in dynamic geometry allowing them to understand how the rectangle is constructed and what happens when $\mathrm{C}$ is dragged. We asked them whether B and D are free points. Half of the class answered positively and explained that these points "are able to move”. See also Laborde, Kynigos, Hollebrands \& Strasser (2006, p. 285). 
they create $F, G$ and $E$ as free points in the plane or on a segment, visually making a square DEFG, but this square does not resist when moving E on [CD]. Obviously, here this analysis of instrumental needs has not been done by the teacher, the weak students needing much time and strong help to achieve this task. Using the trace of a point $\mathrm{K}$ of $x$-coordinate $\mathrm{DE}$ and $y$-coordinate the sum of the areas in sub-task 2 could help reinforce the idea of graph, but here, with no special attention from the teacher, the task is difficult and not meaningful. Weak students do not succeed and high achieving students do not see the trace as a graph of a function.

Forming the algebraic expression is at stake in sub-task 3 . We observe that weak students simply do not understand the task, while high achieving students succeed but do not see a real purpose. For a meaningful transition between dynamic geometry and algebra, students would need some guiding thread. This thread should be the idea of function, existing as a dependency between geometrical objects, dependency between measures, and finally algebraic function. However, the dependency between the area and the position of point $\mathrm{E}$ is not at stake, the independent variable is imposed to students and a function is mentioned only in task 3 , and thus students, high as well as low achievers, experience this task as an abrupt and meaningless switch to skill oriented algebra. The teacher conducts no classroom discussion on dependencies, on the choice of variables and on the relation with the algebraic function. She rather concentrates on a technique of proof, oriented on properties of expression and a special algebraic manipulation technique.

Our view is that, before switching to the usual algebraic space, students should have worked on functions in two other spaces. One is pure dynamic geometry: when moving E to the right, the square visibly grows bigger and the triangle smaller; this could be an opportunity to make students feel the need to quantify in order to study variations of the area of the "whole" shop-ensign. The second working space deals with this quantification", especially building measures that could be involved in the definition of a function: in this working space, exploring dependencies between measures, students could approach a solution, but without the affordances and constraints brought by formalization. Working in these spaces and discussing their limits would have allowed a more meaningful transition to the algebraic space. In this last space, a validation congruent with the work on measures (i.e., using properties of variations for quadratic functions observed on graphs, rather than completing the square) would have been more relevant for connecting algebraic work and work in the previous spaces.

This classroom situation illustrates the narrow treatment of functions by upper secondary teaching that we mentioned above. Optimizing a geometrical quantity is an emblematic type of task in the domain of function. It could provide the opportunity to work on functions in three spaces, and make meaningful connections. Unfortunately, work in the algebraic space is privileged and disconnected from the work in dynamic geometry which is used only as a motivation.

\section{Functional working spaces and the Casyopée software}

The above example of a classroom situation helped us to introduce three spaces for working on functions, and to stress the importance of providing students the opportunity of real work in each of these spaces, as well as to make connections between these ${ }^{6}$. We develop here the idea of connected functional working spaces that arise in

\footnotetext{
${ }^{5}$ About quantification and its importance in students' understanding of functions, see Thompson (2011).

${ }^{6}$ In addition, considering functions in "real world situations" would suppose a space where students could work in a non-mathematical space, for instance around a physical device involving a mechanical dependency. See Lagrange (2013) for
} 
our group while progressing in our understanding of the challenges brought about by the teaching/learning of functions. We also present some aspects of the Casyopée software, one of the resources whose design is carried out by our group as a result of this progress.

In the dynamic geometry working space, functions exist in a figure, with free points and dependent constructions. No quantification is at stake. Students experience dependency between objects, and a first approach to optimization. We stressed above that, in the above example, while dragging the free point $\mathrm{E}$, students could observe and discuss how the shape of the shop-ensign is transformed. Artefacts are primitives of construction, such as creation of parallel or perpendicular lines, and intersections of those, that allow dynamicity. Rules are geometrical principals governing constructions and also features specific to dynamic geometry, especially the asymmetrical relationship between free points and dependent constructions.

In the second space, the objects are measures in relation of dependency. The artefacts deal with prealgebraic symbolism, that is to say formulas expressing geometrical measures by way of algebraic operations and elementary measures. The rules deal with dependencies relative to a moving object: a dependency has to be expressed by way of quantities that are measurable attributes of the object; one quantity (the independent variable) has to depend univocally on the object. These rules are at the very heart of the idea of function.

In the third space, in contrast to the two previous spaces, objects, artefacts and rules exist in the "traditional" paper/pencil school culture: analytic functions, algebraic notation with literals for the independent variable and parameters, graphs and tables, and transformations conserving equivalence. However, as we saw in the analysis of the classroom situation, the idea of function and the link with other spaces can be overlooked because of an overemphasis on algebraic manipulation. In this space, implementing optimization problems should rather help students to reflect on different formulas for a function, their equivalence, and how they express a dependency. In this approach, algebraic transformations (factoring, expanding, differentiating...) exist as artefacts used for a given purpose in view of determining properties of a function.

The Casyopée software is designed to provide artefacts adequate for each of the above working spaces in three main windows (figure 2). The geometry window has generic dynamic geometry features, providing tools for dynamic construction. Free point in the plane is distinguished from free point on an object and from point depending on one or more free points, for instance an intersection of objects constructed from free points. The "geometric calculation tab" allows forming and numerically exploring "pre-algebraic" formulas representing geometrical quantities; it also offers the possibility of selecting two formulas, one for the independent variable, and the other for the dependent variable. In cases where these formulas represent measures that are in functional dependency (like for instance the distance AE and the sum of areas, in the example above), Casyopée is able to compute symbolically an analytic formula and a domain, thus creating a function which is exported into the symbolic window. Otherwise it issues some explanation for the impossibility. Thus, what is really at stake is the selection of measures in functional dependency and the forming of pre-algebraic formulas for these measures. The symbolic window is designed to be consistent with the view we expressed above, of helping students to consider different equivalent formulas for a function and to use transformations as artefacts to study functional properties. It also offers features similar to symbolic graphing calculators (evaluation of expressions, graphs and tables)

an example. Here, for the sake of simplicity, we will keep the type of task of geometric optimization as an object for reflection, and then restrict ourselves to three spaces. 


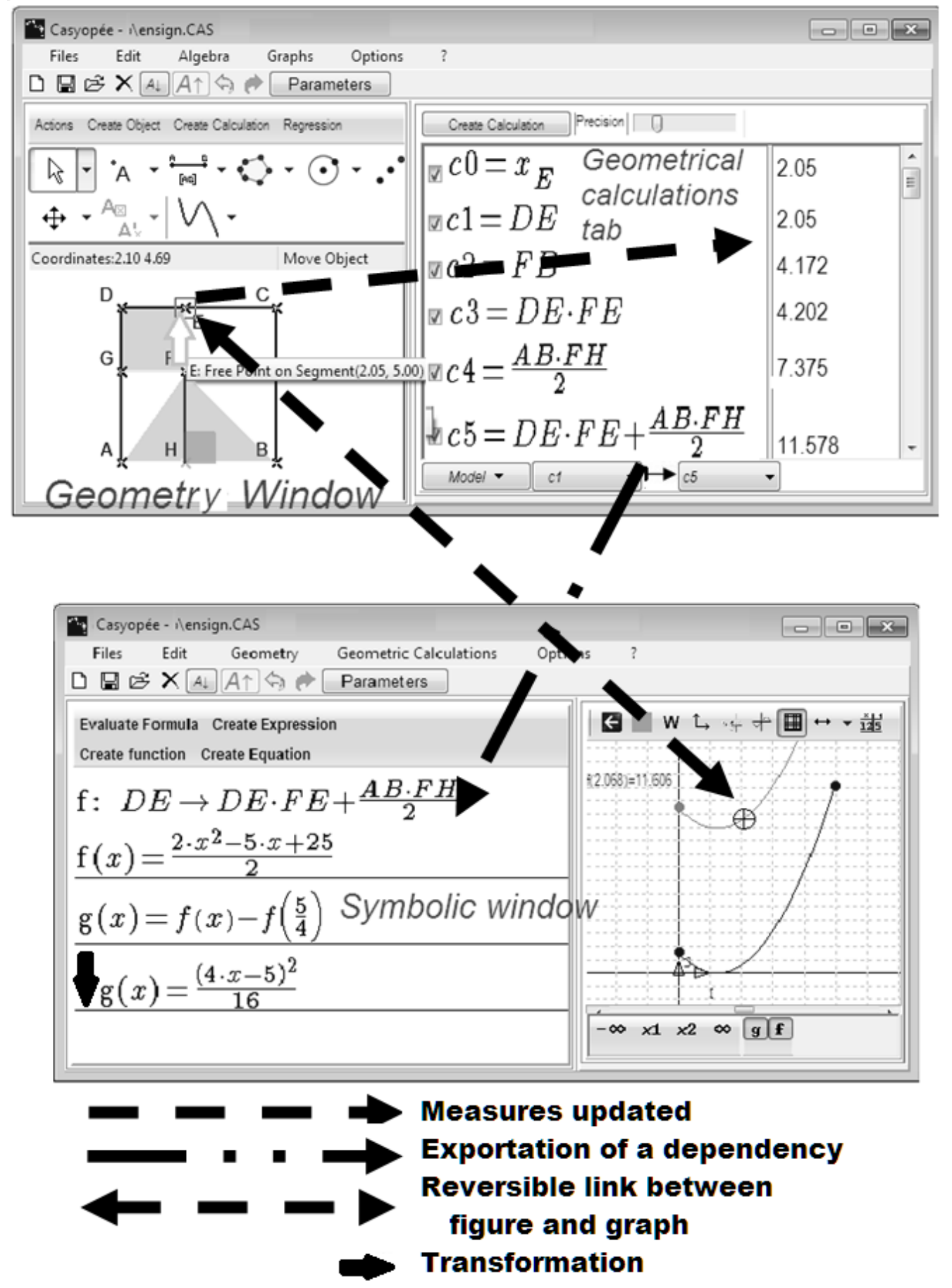

Fig. 2 Casyopée's main windows and the links between them

While the three windows are clearly distinguished, the design helps connections between working spaces. Numerical values of measures created in the geometric calculation tab are displayed and continuously updated when a free point is dragged in the geometry window. The exportation of a dependency from the geometric calculation tab to the symbolic window was decided in order to allow a flexible transition from measures to algebra: students can choose freely relevant measures and change their choice easily if necessary. There are also links between the geometry window and the symbolic window. When a point is dragged in the geometry 
window, the graphs and the formulas are updated. If this point is involved in the definition of the independent variable, a trace is moved on the graph of the function. This link is reversible.

\section{The design of a classroom situation}

We consider now more directly our hypothesis about suitable teaching situations organizing students' work in various non algebraic and algebraic working spaces, and connecting these. We consider again a geometric optimization situation similar in its aims to the "shop-ensign" that we analysed above. It is also a situation for a one hour session at $10^{\text {th }}$ grade. The principles of design are very different and can be expressed with regard to students' work in the three spaces.

1. The dynamic geometry construction and exploration have to be simple, in order that students understand easily the dependency between geometrical objects, even with a limited understanding of this type of construction.

2. The question has to be expressed in pure geometrical terms, in order that students discover the need for introducing a dependent quantity, as well as an independent one.

3. The situation has to emphasize this quantification and the properties of the resulting function, rather than algebraic manipulation.

The choice is then to consider a parabola of equation $y=f(x)$, and a fixed point A not on this parabola, and not on its axis. The question is then to find a position on the parabola "as close as possible" to A (Fig. 3).

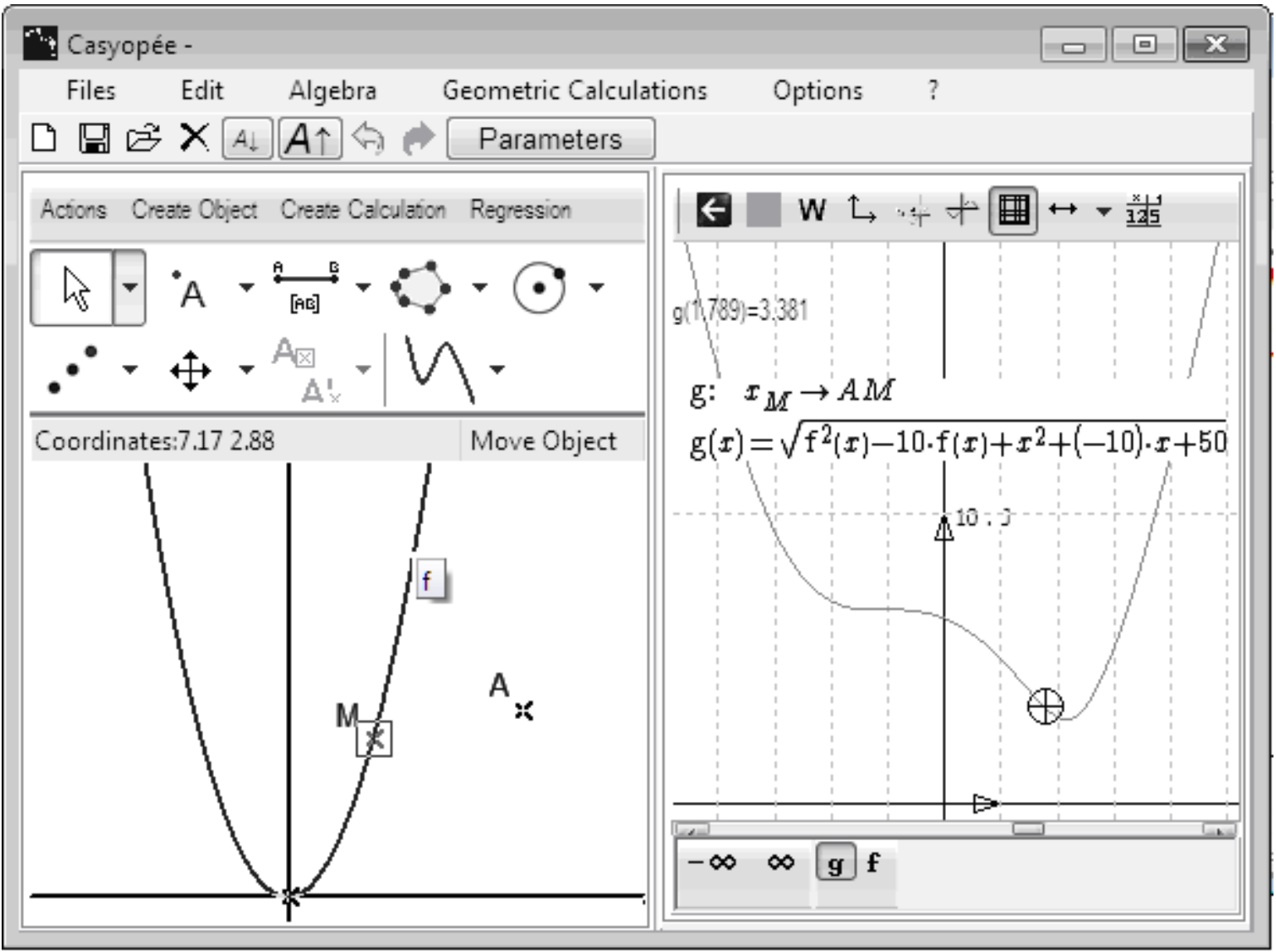

Fig. 3 The dynamic geometry figure, and the formula and graph of the function in the parabola situation 
The situation is then designed in three phases, corresponding to the challenges identified by Lagrange and Psycharis (2014): covariation, independent variable and symbolism.

Phase 1. In the dynamic geometry space, students have to understand the need for introducing a free point $\mathrm{M}$ on the parabola, and then to locate a closest position visually. To be more accurate, students should consider distance $\mathrm{AM}$ and its numerical variations by moving $\mathrm{M}$ on the parabola, and then understand a co-variation between the point $\mathrm{M}$ and the distance. This phase will be carried out by students working with Casyopée.

Phase 2. Introducing this phase, the teacher will stress the need for creating a function in order to have a more accurate value of the minimum. Actually students have been initiated to the graphical exploration of a function given by a formula, and this is for them at this level the best way to explore variations. The critical point here is the identification of an independent variable. Mathematically it is simple, since a free point on a parabola whose axis is the $y$-axis, is parameterized by its $x$-coordinate. However we expect that for students this quantification, passing from the position of a point to a numerical value representing this position, will not be obvious. Then this phase will be a collective discussion directed by the teacher.

Phase 3. Students, working again on computer, will enter the independent and dependent variables into the modeling menu of Casyopée and export a new function into the symbolic window. They will exploit its graph to get a more accurate minimum, a technique that they already know. In order to work on symbolism, students will have also to interpret the formula calculated by Casyopée.

Lagrange (2013) reports on a classroom implementation of this scenario. It shows how students work in each space, and how the teacher helps the transition between spaces by specific mediations.

At the beginning of the first phase, students work on exploration in the dynamic geometry space, creating and dragging a free point $\mathrm{M}$ on the parabola, and observing. This is generally not accurate, for instance students locate a point $\mathrm{M}$ on the perpendicular to the axis passing through $\mathrm{A}$. The teacher asks for a first quantification, referring to Casyopée: "How could we use the software? ... What could we ask it to calculate?" Students understand the interest of a measure to pass from "visually close" to "minimum distance".

This brings them, in the second phase, to the measure space, Casyopée providing the artefacts of the geometric calculation tab. The teacher introduces the problem of quantifying the independent variable: "in order to get a better approximation, we need to define a function whose value is AM... but depending on what...?" The students propose $\mathrm{M}$ and she replies: " $\mathrm{M}$ is not a variable... When you move $\mathrm{M}$, it depends on what? What gives the position of a point?" Then the students propose "the coordinates" or the " $x$-coordinate and $y$-coordinate". She objects: "I have to choose, which one?" Most students choose the $y$-coordinate because visually, this coordinate seems more in relation with the position of $\mathrm{M}$ on the parabola. She objects again: "the $y$-coordinate... then... if I have to locate a point on the curve, what should you give to get the right position? If you ask me for the $y$ coordinate 4..." Students recognize that there are two points for one value of the $y$-coordinate, and propose the $x$ coordinate. The teacher insists: "Yes, if you say, the point is on the curve, and I know the $x$-coordinate, then I know the position of the point...Then you can characterize the position by the $x$-coordinate." Students who tried exporting a function with Casyopée, using the $y$-coordinate and then the $x$-coordinate, confirm that the first choice was not successful.

In the third phase, after graphing the function obtained by exportation, students carry out a graphical exploration, connecting a technique that they already know with the problem of optimization. The teacher shows the reversible link between trace on the graph and position of $\mathrm{M}$ on the parabola, helping students to better 
understand this connection. Interpreting the formula calculated by Casyopée is not easy for the students, because they are not familiar with the technique of expressing the coordinates of a point on a curve, and a distance in coordinate geometry, although it was already taught. They nevertheless recognize key elements in the formula like $f(x)$ denoting the $y$-coordinate of $\mathrm{M}$, and the square root denoting the calculation of a distance.

It seems to us that, although the session is short, the students are aware of working in three different spaces, with different means of representing, treating and interpreting. Students understand dependency between a geometrical object and a property of the figure in the first space, and they are familiar with reading properties on a graph in the algebraic space. The measure space, and particularly the need for expressing a dependency between two measures are new for them. Working in this space is essential for connecting from the figure in the dynamic geometry space to the graph and formula in the algebraic space.

\section{Student's Geneses}

As explained in the introduction we want to take advantage of the identification of functional working spaces, in order to study students' geneses involving particular spaces, that is to say how they build suitable personal spaces. As also explained, our hypothesis is that these geneses necessarily occur in the long term, and we use for that a study of students over two years. The method was to consider the same class of scientific students at $11^{\text {th }}$ and $12^{\text {th }}$ grades and to focus particularly on two students working as a team. The teacher was a member of the Casyopée group. A special observation of the team was carried out by way of screen and video recordings, and of semi-directed interviews. We consider here three milestones: one observation at a key time in each year, and an interview at the end of the second year.

The first observation took place in January of the first year ( $11^{\text {th }}$ grade) on the occasion of the concluding session of an experiment consisting of six sessions. The first three sessions focused on capabilities of Casyopée's symbolic window and on quadratic functions. In other words, this part was an introduction to an algebra space where Casyopée provided artefacts, and rules were related to algebraic knowledge regarding quadratic functions. The next two sessions aimed at consolidating students' knowledge of geometrical situations and at introducing them to Casyopée's other windows. The central task was to build geometric calculations to express areas and to choose relevant independent variables to express dependencies between a free point and the areas. This part can be considered as an introduction, both to the dynamic geometry and to the measure spaces. It puts emphasis on measures that are new for students. The third part consisted of one concluding session that will be considered here as a first observation of students' geneses. Students had to solve the first problem in figure 4.

The second observation took place in January of the second year ( $12^{\text {th }}$ grade), was about students solving the second problem in figure 4. Meanwhile the class worked on similar problems, roughly one session every month and during these sessions, the two students interacted with the observer. The interview was carried out at the end of the second year, before the baccalauréat ${ }^{7}$. Meanwhile students worked on functional problems

7 "Much like British A-levels or European Matura, the baccalauréat allows French students to obtain a standardised qualification, typically at the age of 18 . This then qualifies holders to (...) go on to tertiary education.” (https://en.wikipedia.org/wiki/Baccalauréat). The students observed here pass the scientific baccalauréat involving a set of subjects among which mathematics has the heavier weight. 
adequate for preparing this exam (and then mainly algebraic) and used Casyopée as a resource for personal work ${ }^{8}$.

\begin{tabular}{|c|c|}
\hline $\begin{array}{l}\text { Text: Consider a triangle } \mathrm{ABC} . \mathrm{A}(-\mathrm{a} ; 0), \mathrm{B}(0 ; \mathrm{b}) \text { and } \\
\mathrm{C}(\mathrm{c} ; 0), \mathrm{a}, \mathrm{b} \text { and } \mathrm{c} \text { being three parameters. Find a } \\
\text { rectangle } \mathrm{MNPQ} \text { with } \mathrm{M} \text { on }[\mathrm{OA}], \mathrm{N} \text { on }[\mathrm{AB}], \mathrm{P} \text { on } \\
{[\mathrm{BC}], \mathrm{Q} \text { on }[\mathrm{OC}] \text { and with maximum area. }}\end{array}$ & $\begin{array}{l}\text { Problem } 2\left(12^{\text {th }} \text { grade }\right) \\
\text { Text: Consider point } \mathrm{I}(0 \text {; a), a being a parameter and } \\
\text { point } \mathrm{A} \text { of coordinates (10; } 0) \text {. M belongs to segment } \\
\text { [OA], } \mathrm{N} \text { is on the parallel to the } \mathrm{y} \text {-axis passing by } \mathrm{A} \\
\text { and the triangle IMN is rectangle in } \mathrm{M} \text {. When } \mathrm{M} \text { is in } \\
\mathrm{A} \text {, then } \mathrm{N} \text { is also in } \mathrm{A} \text {. The problem is to find the } \\
\text { position of } \mathrm{M} \text { to maximize the area of the triangle. } \\
\text { Hint of a solution: for values of a greater than } \frac{10}{\sqrt{3}} \text {, the } \\
\text { function is decreasing and then the maximum is for } \\
\mathrm{M}=\mathrm{O} \text {. For other values, there is a local maximum for } \\
\text { a position of } \mathrm{M} \text { inside the segment. This maximum is } \\
\text { the absolute maximum for a lower than } 5 \text { (figure } 2) \text {, } \\
\text { otherwise the maximum is for } \mathrm{M}=\mathrm{O} \text {. For a }=5 \text {, there } \\
\text { are two maximums, one for } \mathrm{M}=\mathrm{O} \text { and the other at the } \\
\text { middle of [OA]. }\end{array}$ \\
\hline
\end{tabular}

Fig. 4 The problems in the two observations

In each working space, we analyse the tasks and how students progressed from one year to the next.

In the dynamic geometry space, the figure in both problems is defined parametrically. Consequently, the dynamic figure includes points defined by parametric coordinates, as well as a free point on a segment and then students have to distinguish between the two notions. Dependent points are to be constructed, as in the "shopensign" situation. The first year, the students took much time constructing the variable rectangle. They first built a "soft" rectangle, that is to say that the quadrilateral they built was perceptively a rectangle, but did not resist a variation of the figure by animation, dragging a free point or changing the value of a parameter. They had difficulty in distinguishing between a free point in the plane and a free point on a segment. In spite of the feedback of the software and of the help of the observer, they were slow to correct. The second year, mistakes in using dynamic geometry still occurred, but students easily corrected the errors by themselves. It means that in this space, becoming aware of the dependency between objects was not obvious but progressed, thanks to sustained work in this space. Working on dependencies from geometrical situations actually puts at stake this fundamental aspect of dynamic geometry. Our interpretation is that these students did not meet in previous classes the constraint of constructing a figure whose properties resist animation. More generally, we assume that in many classrooms, in spite of the official demands, students do not have to use dynamic geometry by

\footnotetext{
${ }^{8}$ More data about this study is provided by Minh (2012b).
} 
themselves, or have mostly to draw "static" figures". The outcome is that teaching has to take particular care of students' genesis relative to the dynamic geometry space, providing opportunities of working on really dynamic figures and discussing the resulting dependencies.

In the measure space, finding a formula for the dependent variable is not difficult. In both problems, several choices are possible for the independent variable, and, especially for the first problem, this choice has an influence over the complexity of the formula of the algebraic function: if students choose a length on the $x$-axis representing the position of $\mathrm{M}$, the formula is less complex as compared to the formula resulting from the choice of a side of the rectangle. Numerical exploration of these variables is to be made more carefully in the second problem, since variations depend on the parameter. The first year, the students confused the creation of a dependant variable representing the area with the choice of an independent variable and hesitated on the choice. They did few numerical explorations. They did not understand by themselves the need to choose an independent variable and to export the dependency as an algebraic function. The second year, although the exploration is more complex, students explored a lot of different cases and values of the parameter. They commented, using the relevant functional language: "growing", "decreasing"... There was also a much better understanding of the process of exporting the function beyond the corresponding artefact of Casyopée. Particularly, as shown by Minh (2012a), students identified the choice of variables in the measure space as the key action. This means that sustained work is useful in this space for an adequate understanding of functions.

In the algebraic space, the techniques for proving the variations are different in the two years, following the progress of students' learning in calculus. In the first problem, students are expected to use knowledge about quadratic functions: their graph as a parabola, and a formula giving the abscissa of the vertex of the parabola, from coefficients of an expanded form of the function. In the second problem, students are expected to differentiate the function, to obtain a quadratic function and to compute the zeros (figure 4). These calculations are classical. However the formulas are parametric and this aspect introduces some complexity, so students were expected to use Casyopée to feel more confident. The difficult part in the second problem is to interpret the zeros, because depending on the parameter, they can be real or complex, or they can be inside or outside the interval of definition of the function. In the first year, after recognizing a parabola for the graph of the function calculated by Casyopée, students did not know how to use their previous knowledge about quadratic functions. With the help of the observer, they remembered a formula for the abscissa of the vertex. The resulting expression was complex, but could be easily simplified by Casyopée. In spite of this, students dealt only with a numerical case, considering the current values of the parameters. Finally the students conjectured the optimal position for a generic triangle in the geometry window, but could not prove it in the symbolic window. The second year, students proposed directly a procedure using the derivative. They used smartly Casyopée's transformations such as "expanding" and "factoring" in view of a goal. They used the sliders in Casyopee to change the value of the parameter, in order to study the different cases. In short, they built a suitable personal algebra working space. One could object that this personal space is closely linked to Casyopée's artefacts. However, this space was useful in the preparation of the baccalauréat. We assume that this comes from special strategies of the teacher and we keep the elucidation of these strategies for further work.

\footnotetext{
${ }^{9}$ Some evidence supporting this assumption is provided by Lagrange \& Caliskan (2009) from a study of textbooks and classroom practices in France.
} 


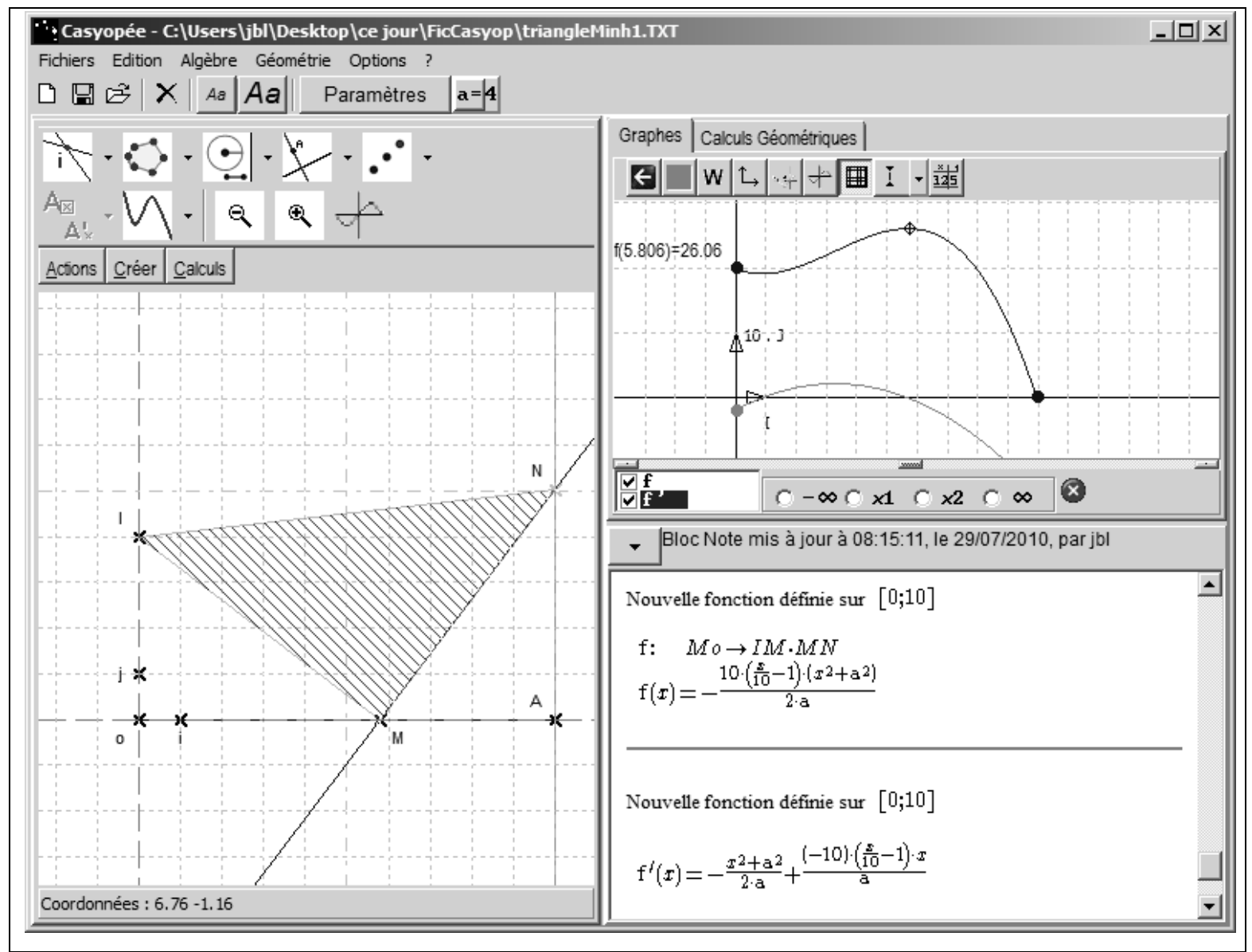

Fig. 5 Problem 2 figure and graph of the function and derivative for the case $a<5$

As reported by Minh (2012a), the interview at the end of the second year shows that the students also connected the work in the three spaces in a "process" contributing to the elucidation of a function, for instance when they declared: "to perform all the process is great: constructing the figure, table of variation, calculation of the derivative... We have the algebraic and geometrical sides together... We see better how a function 'reacts', it is convenient and interesting”.

\section{Teachers' geneses}

Like the preceding section, this section is about geneses concerning functional working spaces, but with regard to teachers, with the same hypothesis that theses geneses necessarily occur in the long term. We use data from a project that aimed at studying how innovative software like Casyopée can be disseminated to "ordinary" classrooms. This project brought together two teachers that had been involved in the design of Casyopée, named "experts" in this paper, and six other teachers named "participants" in this paper. The experts were members of the Casyopée group since the beginning. The participants were relatively experienced in the classroom use of technology but not involved in the history of Casyopée.

The idea was to use classroom scenarios as means for communicating between experts and participants. The participants would propose uses corresponding to their needs and ask the experts for their advice and support. The scenarios would be built in order to be proposed to all potential users, and thus contribute to the dissemination. Researchers, experts and participants met 14 times during 2 years in 3 hour sessions. Eight different scenarios were created and each was experimented in several classes. The choice was to analyze 
objective elements accounting for communal work, especially the video recordings of the meetings. The recordings were coded in a video analysis database and split into about 90 clips. We consider here that preparing, experimenting and presenting classroom situations with Casyopée implied for participants to make sense of new working spaces for their students, characterized by the new artefacts provided by Casyopée, but also influenced by the underlying conception of the work on functions, and then to build personal working spaces adequate for teaching.

Because the participants were already experts in classroom use of dynamic geometry, we consider here only the algebra and measure spaces. We do the analysis by using interactions recorded in the clips, completed by data related to the classroom scenarios produced by the group.

In clips dealing with the measure space, it appears that the participants had implemented before geometric optimization situations in their classes, similarly to how the teacher did for the shop-ensign problem reported above. They were unsatisfied because of the algebraic manipulation work, which they considered not relevant during the resolution. They were then tempted by classroom use of the geometrical calculation tab. However they encountered difficulties. In the second meeting for instance, a teacher complained that she could not use exportation for a problem she used to propose to her students (fig. 6). She considered two free points in the plane $\mathrm{A}$ and $\mathrm{B}$ and a free point $\mathrm{M}$ on the segment $[\mathrm{AB}]$. After that, she wanted students to export a dependency into the symbolic window, in order to solve the problem algebraically. She chose AM as the independent variable and the calculation $\frac{A P^{2}+M Q^{2}}{2}$ as dependent variable. But Casyopée replied that the calculation AM is dependent on more than a free point, and exportation was unsuccessful. The teacher expected a function like $x \rightarrow \frac{x^{2}}{4}+\frac{(A B-x)^{2}}{4}$. Actually, Casyopée handles functions of one variable. It is also possible to create parameters and then to consider families of functions. Thus in any situation in which several variables are involved, one variable has to play the role of the independent variable and the others have to play the role of parameters. In the above situation, a consistent approach is to define the two points A and B as coordinated points, depending on a parameter representing the distance $\mathrm{AB}$. For instance $\mathrm{A}$ might be at the origin and $\mathrm{B}$ might be $(0 ; \mathrm{a})$ and the function would be $x \rightarrow \frac{x^{2}}{4}+\frac{(\mathrm{a}-x)^{2}}{4}$. This is a constraint of a functional working space centered on one variable functions when dealing with objects depending on several variables. Understanding this constraint is not essential for students who work on situations prepared by the teacher, but the teacher has to deal with this when preparing a situation and he or she needs a deeper understanding of dependency. During the meetings and the experimentations of scenarios, it seems that the participants integrated this constraint: when presenting the scenarios elaborated in the project, they often regretted that "the teachers do not understand this very simple fact that for modeling into a function, you have to restrict to a single free point". 


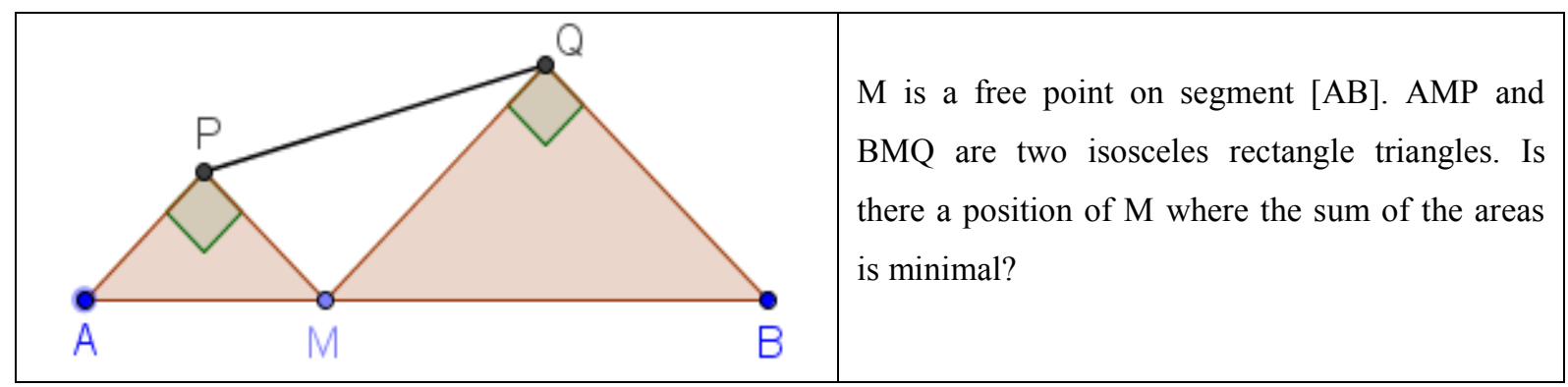

Fig. 6 An optimization problem proposed by a participant

We characterize the participants' genesis relative to the algebra space first using clips recording interactions that dealt with the participants' expectations and interrogations concerning Casyopée's symbolic window that these teachers identified as providing Computer Algebra (CAS) tools. Participants thought initially that CAS would be particularly useful for problems about functions in real world situations often implying complicated algebraic calculations. This idea was linked with their desire to offer students realistic problems, and with views that CAS should be used as scaffolding for students with weak abilities in algebra. That is why they proposed situations resulting in very complex expressions that CAS had difficulties handling. This is because CAS relies on algorithms that are generally not deterministic: for instance, it happens that two expressions are mathematically equivalent but also that none of the algorithms can recognize the equivalence, a phenomenon that Elbaz-Vincent (2005) points out as the "decidability limitation" inherent in CAS.

It took time for these teachers to realize how the CAS' decidability limitation influences the classroom situations. Scenarios had to be carefully adapted in order to articulate CAS and by hand calculation. Then participants realized the actual potential of CAS for teaching/learning: it does not miraculously handle everything, but it can help when one understands its potential and limits, and takes advantage of these for specific situations. At the end of the project, from a participant's proposition, a classroom scenario was designed and experimented, where students had to coordinate the use of Casyopée for calculations that they were not confident to perform by themselves, and by hand simplifications that could not be achieved by CAS. This means that, while participants understood better the potential of CAS artefacts, implicit rules for their didactic use also evolved.

In order to give insight into the complexity of the participants' evolution concerning the algebra space, we examine another example dealing with techniques of proof. It happened that an expert proposed a scenario where $10^{\text {th }}$ grade students had to conjecture and prove the interval of positivity of a given linear function. He proposed that students conjecture graphically and then use the monotonicity of a linear function that allows a proof congruent with the graphical observation and supported by Casyopée ${ }^{10}$. Participants agreed about making students conjecture this property on graphs, but objected that monotonicity would have to be proved. This is a summary of the discussion that followed between the expert (E) and participants (Pi).

The expert proposes a proof for monotonicity.

E: My view is to use factoring. Computing $f(u)-f(v)$ and then factoring into $a(u-v)$.

P1 reports that she tried to use factoring to prove the variations of quadratic functions at $11^{\text {th }}$ grade without much success. E objects that an inequation based technique is "rule oriented".

\footnotetext{
${ }^{10}$ There is a special functionality, based on algebraic theorems dealing with functions, that helps building proofs.
} 
P1: Factoring ? When I tried to apply to quadratric functions at 11 th grade... the link between $f(u)-f(v)$ and $\mathrm{u}-\mathrm{v}$ is perhaps more visible... but, but.. I finished by doing only inequations...

E: OK, applying rules...

Another participant (P2) objects that a technique based on monotonicity is very new to $10^{\text {th }}$ grade students and wonders how students could be engaged in a proof. P1 stresses that the teacher will have to guide them strongly and P2 confirms that proving the variations of quadratic functions at $11^{\text {th }}$ grade is very hard.

P2: We must start from what they (the students) know, otherwise ... My question, how will they compare $f(u)$ and $f(v)$ ?

P1: You will tell them (students) to calculate $f(u)-f(v)$. You will say : "to compare $f(u)$ and $f(v)$, calculate $f(u)-f(v)$ ". You will tell them.

P2: For quadratic functions at $11^{\text {th }}$ grade, calculating $f(u)-f(v)$, good students understand, but... it is hard, very hard...

E suggests that, since rules about inequalities have been used without proof, the monotonicity of linear functions could also be admitted without proof. Actually Casyopée proposes the variation property of a linear function (growing, decreasing) based on the sign of the $x$-coefficient. Students could then make the link between this sign and the variation property by graphical observation. The participants discuss the relevance of introducing a new technique, from their experience of teaching $10^{\text {th }}$ graders something already taught at $9^{\text {th }}$ grade.

E: Rules learnt at 9th grade about inequalities are also artificial (not proved).

P2: The problem is to prove again at $10^{\text {th }}$ grade something they manipulated and remanipulated at $9^{\text {th }}$ grade...

E: What do they really remember (of what they did at $9^{\text {th }}$ grade)?

P3: They don't really remember. I did it (when teaching at 9th grade) and now I do the same at $10^{\text {th }}$ grade... I have the impression to do about linear functions at $10^{\text {th }}$ grade what I was doing before at $9^{\text {th }}$ grade...

Finally, the participants seem ready to adopt the new technique but are concerned with how students could make connections between the two techniques.

P2: Then it is perhaps not a problem to change...

P3: Yes and no, they (the students) should make a link...

The participants' reluctance towards the technique proposed by the expert has complex motivations. First, this is a functional technique, (i.e. technique based on properties of functions), as compared to inequations, and the participants prefer to keep it for more advanced students and for non-linear functions: at $11^{\text {th }}$ grade, a complete proof by factoring seems to them relevant, although not obvious for most students. Second, the participants like to keep the proof by inequations for $10^{\text {th }}$ graders, even when it has already be taught at $9^{\text {th }}$ grade, because they are concerned that these students would forget algebraic rules learnt before. This discussion occurred at the end of the first year and, although the participants seem to consider more favorably the proposition after the discussion, it has not been put into operation as a classroom scenario.

The participants' resistance to techniques of proof based on functional properties indicates that, as a difference from the expert's, their new personal functional algebra space is in competition with an "old" equation based space. This can be put into relation with the problem of the coordination between (in)equation based algebra taught before $10^{\text {th }}$ grade, and the functional approach at $10^{\text {th }}$ grade. Curricula encourage switching to functions, leaving to the teachers the difficult task of coordinating these approaches (Kieran 2007). This task has been achieved by the experts along the years in the Casyopée group, building a suitable functional space; in this 
space the technique of proof is congruent with the graphical conjecture, taking advantage of students' new knowledge about functions. It was not the case of the participants even after two years of interactions.

Finally, we note also that the "closest point on the parabola" scenario exposed and analyzed above was a common production of participants and experts after a proposition of participants at the end of the project: the participants insisted on designing a situation for $10^{\text {th }}$ graders minimizing the instrumental needs in dynamic geometry, as well as the algebraic manipulations, in order to focus on the choice of variables by the students. Implicitly, the teachers recognized the necessity for students of situations bringing forth the question of the variables involved in a function and then the central role of the measure space. The necessity of connecting the spaces is also implicitly recognized through this situation; the teacher observed in the implementation of the scenario reported above was one of the participants and we saw how she insisted on the coordination between the works in the three spaces.

\section{$7 \quad$ Summary and perspectives}

We presented this paper as a continuation of previous work aiming at remedying a narrow treatment of functions by upper secondary teaching, especially taking into consideration the fact that functions make sense because of their occurrence in many dissimilar settings, and then the need for analysing each of these settings as a functional working space. We identified geometric optimization as an "emblematic" type of task and analysed a classroom implementation, pointing out problems resulting from the fact that no working space had been prepared by the teacher for students' tasks in dynamic geometry and measures. Thus the situation gave priority to the algebraic manipulation work, poorly contributing to students' understanding of functions. We specified a dynamic geometry space, a measure space, and an algebra space, and indicated specific functionalities of Casyopée bringing artefacts in each space and means for connecting the spaces. We examined then the functionality of this framework for implementing and analyzing classroom situations and for analyzing students' and teachers' evolution concerning functions, in terms of geneses related to each space. We gave an example where considering students' activity in each space and connections between spaces, allows the development of principles of design for a classroom situation of geometric optimization, to assess their efficiency and to highlight differences from the situation analyzed before.

In previous papers, for instance by Lagrange and Psycharis (2014) or Minh (2012b), the main theoretical construct was a grid of students' activities on three levels: (1) Covariation and dependency in a physical system (2) Covariation and dependency between magnitudes or measures (3) Mathematical functions of one real variable. The grid also proposed a classification into types of activity (Enactive-Iconic, Algebraic...). This is of course compatible, but organizing and analyzing working spaces goes beyond classifying activities: it is specifying the settings in which students develop these activities and make sense of these. In addition, the grid insists on a similarity between activities at each level, whereas each space has its specific artefacts and rules. Finally, the grid seems to imply a more or less standard path in the activities from physical settings to algebra. Considering working spaces provides a less rigid view of students' work about functions. For instance, the Casyopée group recently elaborated and tested a classroom scenario based on several models of a physical object (the main cable in a suspension bridge): a local physical model involving tensions and the first Newton law, a local mathematical model based on the relationship between tensions and positions of two consecutive points on the cable, a computational discrete model calculating iteratively the coordinates of points on the cable together 
with the tensions, and finally a continuous global model using calculus (see http://casyopee.eu). Each model is subject to specific scientific work and for students, corresponds to a specific space. The spaces are explored in parallel by groups of students and after that, groups of students work to prepare a synthesis. The outcome is that a diversity of functional spaces, not limited to the three considered in this paper, may be organised and investigated by students in a nonlinear way. This situation also implies a wider variety of artefacts: films, concrete models, paper/pencil work and a programming environment. It will be an opportunity, in further work, to explore the functionality of the framework beyond the three spaces studied here, and beyond artefacts specific to Casyopée.

The two studies about geneses insist on long term and on particular geneses in each working space. Students' geneses show a gradual construction of personal spaces and connections between these, thanks to sustained work articulating the three spaces. Teachers' geneses are also gradual, in some cases unachieved, and can be seen as the result of the work of creating classroom situations together with experts. Teachers' personal spaces include an understanding of functional dependency and of the use of artefacts as a basis for these situations: in other words they incorporate rules about the constitution of working spaces for students.

Up to now, we used the theoretical framework of "instrumental genesis" (Lagrange, 1999; Minh, 2012a, Minh, 2012b) to study the development of knowledge in situations of use of mathematical software environments. Instrumental geneses share with the geneses introduced in this paper a common basis: they link closely the appropriation of capabilities of computer artefacts and the development of relevant knowledge, mathematical for the students and didactical for the teachers. It seems to us however that geneses relative to particular spaces that we considered in this paper, have the advantage of providing a more analytic view of long term evolution, and of being centred on mathematical work rather than restrictively on artefacts.

As we mentioned in note 1, 'genesis' is used in MWS for denoting processes connecting the epistemological and the cognitive planes. This is certainly different, but 'our' geneses clearly imply cognitive evolution of the subjects and so further work could search to reconcile the two constructs. Also, in the continuity of previous work, this paper favors the artefact component of functional working spaces to the detriment of the rules, or more precisely, of the theoretical reference frame, and then further work should also aim at studying more comprehensively this component in each space.

Acknowledgment: This research was supported by Vietnam National Foundation for Science and Technology Development (NAFOSTED), under grant number VI1.99-2012.16.

\section{References}

Elbaz-Vincent, P. (2005). A Cas as an Assistant to Reasoned Instrumentation. In D. Guin, K. Ruthven, \& L. Trouche (Eds.), The Didactical Challenge of Symbolic Calculators: Turning a computational device into a mathematical instrument (pp. 41-65). New York: Springer.

Halbert R., Lagrange J-B., Le Bihan C., Le Feuvre B., Manens M-C., \& Meyrier X. (2013). Les fonctions : Comprendre la notion et résoudre des problèmes de la 3ème à la Terminale. L'apport d'un logiciel dédié. I.R.E.M de RENNES - Université de RENNES.

Kieran, C. (2007). Learning and teaching algebra at the middle school through college levels. In F. K. Lester Jr. (Ed.), Second handbook of research on mathematics teaching and learning (pp. 707-762). Charlotte, NC: National Council of Teachers of Mathematics, Information Age Publishing.

Kuzniak, A. (2013). Teaching and learning geometry and beyond. In B. Ubuz, Ç. Haser, \& M. A. Mariotti (Eds.), Proceedings of the Eighth Congress of European Research in Mathematics Education (CERME 8), pp. 33-49, Antalya, Turkey. 
Lagrange, J. -B. (1999). Complex calculators in the classroom: theoretical and practical reflections on teaching pre-calculus. International Journal of Computers for Mathematical Learning, 4(1), 51-81.

Lagrange, J. -B., \& Caliskan, N. (2009). Usages de la technologie dans des conditions ordinaires : le cas de la géométrie dynamique au collège. Recherches en Didactique des Mathématiques, 29(2), 189-226.

Lagrange, J. -B. (2011). Working with teachers: Innovative software at the boundary between research and classroom. In B. Ubuz. (Ed.), Proceedings of 35th Conference of the International Group for the Psychology of Mathematics Education, Vol. 3, Ankara, Turkey: PME, pp. 113-120.

Lagrange, J. -B. (2013). A functional perspective on the teaching of algebra: current challenges and the contribution of technology. International Journal for Technology in Mathematics Education, 21(1), 3-8.

Lagrange, J. -B., \& Psycharis, G. (2014). Investigating the Potential of Computers Environments for the Teaching and Learning of Functions. Technology, Knowledge and Learning, 19(3), 255-286.

Laborde, C., Kynigos, C., Hollebrands, K., \& Strasser, R. (2006). Teaching and learning geometry with technology. In A. Gutierrez \& P. Boero (Eds.), Handbook of research on the psychology of mathematics education: Past, present and future (pp. 275-304). Rotterdam, The Netherlands: Sense Publishers.

MEN/DGESCO-IGEN. (2013). Les compétences mathématiques au lycée. http://eduscol.education.fr/ressourcesmaths.

Minh, T. K. (2012a). Learning about functions with the help of technology: Students' instrumental genesis of a geometrical and symbolic environment. In T. Y. Tso. (Ed.), Proceedings of the $36^{\text {th }}$ Conference of the International Group for the Psychology of Mathematics Education, Vol 3, Taipei, Taiwan : PME, pp $217-$ 223.

Minh, T. K. (2012b). Les fonctions dans un environnement numérique d'apprentissage : étude des apprentissages des élèves sur deux ans. Canadian Journal of Science, Mathematics and Technology Education, 12(3), 233-258

Nguyen Thi Nga. (2011). La périodicité dans les enseignements scientifiques en France et au Viêt Nam : une ingénierie didactique d'introduction aux fonctions périodiques par la modélisation. Thèse de doctorat, Université Joseph Fourier et Université Pédagogique d'Ho Chi Minh Ville.

Robert, A., \& Vandebrouck, F. (2014). Proximités-en-acte mises en jeu en classe par les enseignants du secondaire et ZPD des élèves : analyses de séances sur des tâches complexes. Recherches en didactique des mathématiques, 43(2-3), 239-285.

Thompson, P. W. (2011). Quantitative reasoning and mathematical modeling. In L. L. Hatfield, S. Chamberlain, \& S. Belbase (Eds.), New perspectives and directions for collaborative research in mathematics education. WISDOMe Mongraphs (Vol. 1, pp. 33-57). Laramie, WY: University of Wyoming. 